\title{
Metodologias e Escala na Climatologia Geográfica - Técnicas Estatísticas e Visão Multi-Escalar para a Compreensão do Comportamento do Clima
}

\author{
Methodologies and Scale in Geographical Climate - Statistical Techniques and \\ Mult-Scalar Vision for Understanding Climate Behavior
}

Metodologías y Escala en Climatología Geográfica: Técnicas Estadísticas y Vista de Múltiples Escalas para Comprender el Comportamiento Climático

João Paulo Macieira Barbosa ${ }^{1}$

https://orcid.org/0000-0002-8626-5063

Alfredo Borges de Campos ${ }^{2}$

https://orcid.org/0000-0002-7507-1482

Vânia Maria Nunes dos Santos ${ }^{3}$

https://orcid.org/0000-0001-5659-7705

RESUMO: Pretendemos, através deste artigo, apresentar algumas das técnicas aritméticas utilizadas nas pesquisas em Climatologia Geográfica, buscando ao final elaborar um fluxograma orientativo sobre a aplicabilidade de cada técnica segundo o objetivo a ser alcançado pelo pesquisador. Além da técnica estatística, faz-se importante ao pesquisador em Climatologia Geográfica a compreensão da dinâmica multi-escalar do fenômeno climático. Para isso, empreendemos uma breve descrição a respeito da escala espacial nos estudos do clima, apresentando, ao final, um modelo para compreensão multi-escalar da dinâmica atmosférica, iniciando na macro-escala de análise e chegando aos elementos interferentes da dinâmica climática em escala local.

PALAVRAS-CHAVE: Climatologia geográfica. Estatística. Escala. Dinâmica.

ABSTRACT: Through this article, we intend to present some of the arithmetic techniques used in research in Geographic Climatology, aiming at the end to elaborate a guiding flowchart on the applicability of each technique according to the objective to be achieved by the researcher. In addition to the statistical technique, it is important for researchers in Geographic Climatology to understand the multi-scale dynamics of the climatic phenomenon. For this, we undertook a brief description of the

\footnotetext{
1 Doutorando em Ensino e História de Ciências da Terra. Instituto de Geociências da UNICAMP. E-mail: j001862@dac.unicamp.br.

Doutorado em Ciência do Solo e Meio Ambiente (Universidade de Purdue-EUA). Professor do Instituto de Geociências da UNICAMP. E-mail: acampos@ige.unicamp.br.

${ }^{3}$ Doutorado em Ciências: Ensino e História de Ciências da Terra pelo Instituto de Geociências da UNICAMP. Professora do Instituto de Geociências da UNICAMP. E-mail: vmnunes@unicamp.br.
} 
spatial scale in climate studies, presenting at the end a model for multi-scale understanding of atmospheric dynamics, starting at the macro-scale of analysis and reaching the interfering elements of climate dynamics at a local scale.

KEYWORDS: Geographic climatology. Statistic. Scale. Dynamics.

RESUMEN: Tenemos la intención, a través de este artículo, de presentar algunas de las técnicas utilizadas en las investigaciones de Climatología Geográfica, buscando elaborar un diagrama de flujo orientativo sobre la aplicabilidad de cada técnica de acuerdo con el objetivo del investigador. Además de la técnica estadística, es importante para el investigador de la Climatología Geográfica la comprensión de la dinámica multiescalar del fenómeno climático. Para eso, hicimos una descripción breve de la escala espacial en los estudios del clima, presentando en el final un modelo para la comprensión multiescalar de la dinámica de la atmósfera, empezando en la macro-escala de análisis y llegando en los elementos que interfieren en la dinámica climática en escala local.

PALABRAS CLAVE: Climatología geográfica. Estadística. Escala. Dinámica.

\section{INTRODUÇÃO}

As pesquisas climatológicas são realizadas segundo paradigmas em torno do conceito de Clima. Várias metodologias podem ser aplicadas no estudo do clima, a depender do objetivo específico da pesquisa e da verificação das hipóteses levantadas. Uma grande questão nas pesquisas dentro da Climatologia Geográfica refere-se à dificuldade de mapeamento de cenários que são incertos. Muitos estudos se utilizam de longas séries históricas para a identificação de tendências futuras. Esses estudos são caracterizados pela aplicação de técnicas estatísticas em longas séries temporais sobre os elementos do clima, buscando compreender sua variabilidade e sua tendência.

Os recursos técnicos à disposição dos pesquisadores, tais como o Sensoriamento Remoto, as Imagens Orbitais, Sistemas de Informação Geográfica e outros, produzem inovações metodológicas. A Climatologia Geográfica utiliza-se amplamente dessas contribuições, porém sem abandonar procedimentos clássicos, como os estatísticosquantitativos, desde que estes se mostrem adequados ao tema investigado. Como em outros setores da Geografia e nas demais ciências, a opção entre as diferentes propostas metodológicas está vinculada necessariamente às escalas de análise, que influenciam nas opções das diversas técnicas matemáticas.

A confluência entre alguns desses recursos técnicos e a estatística clássica permitiu o surgimento de um novo modelo de análise, onde a componente espacial (localização) surge como atributo fundamental dos elementos geográficos, constituindo a geoestatística, como nova possibilidade para a pesquisa com variáveis climáticas. Seus fundamentos se dão 
através da Interpolação Espacial e Simulação (SOARES, 2016), ambos amplamente usados nos estudos da Climatologia Geográfica.

O primeiro fundamento nos fornece a possibilidade de estimativas espaciais a respeito da distribuição de elementos do Clima em áreas onde a ausência dessa informação se faz preponderante; o segundo fundamento relaciona-se com o estudo de cenários, quando, através de técnicas estatísticas apropriadas em correlação com o atributo espacial da variável, permite ao pesquisador vislumbrar condições pretéritas e futuras do elemento climático (precipitação, temperatura...) ou do próprio Clima.

Frente a todas essas possibilidades de análise, pode-se correr o risco de "abusar" do uso da estatística na pesquisa em Climatologia, resultando em um "mascaramento" do comportamento real dos elementos climáticos. O simples cálculo da média de determinado elemento já mascara a ocorrência de episódios extremos. Entretanto, mesmo a abordagem rítmica do clima utiliza-se de índices de participação de massas de ar e de frentes atuantes sobre diferentes áreas do globo terrestre, com vistas à classificação climática de base genética, possível através do cálculo das médias (BARROS; ZAVATTINI, 2009). Sendo assim, podemos falar em metodologias relacionadas ao tipo de tratamento que é dado aos registros climáticos e do uso que se faz desses registros.

A metodologia pode ser mais analítica ou mais dinâmica, a depender de como se trata o conceito de Clima e do que se faz com a informação matemática extraída das técnicas estatísticas. Outra categoria de classificação metodológica das pesquisas climáticas faz referência ao critério da abordagem: quantitativa ou qualitativa.

A metodologia dinâmica normalmente usa um critério de abordagem mais quantitativo, porém também se pode fazer uso do critério qualitativo. Por exemplo, na análise rítmica, a abordagem quantitativa está relacionada ao uso de variáveis aplicáveis a métodos estatísticos e a abordagem qualitativa, de caráter empírico, é a responsável por compreender as características locais e os regimes atmosféricos atuantes no recorte espacial.

Siqueira (2019) lançou mão da combinação das duas abordagens metodológicas, quantitativa e qualitativa, para descrever e analisar o regime pluviométrico na bacia hidrográfica do rio Paraná, bem como os fenômenos meteorológicos de maior incidência sobre o recorte espacial proposto. Isso revela que a individualidade climática só pode ser alcançada quando, num mesmo estudo, a representação qualitativa da sucessão dos tipos de tempo - encadeamento dos estados atmosféricos (dinâmica) - é associada às variações quantitativas dos elementos do clima.

Outra complexidade no estudo dos elementos climáticos faz referência à compreensão da variabilidade da informação. A variabilidade é uma componente fundamental para as pesquisas abordadas sob a metodologia dinâmica e rítmica proposta por Sorre (1951) e 
aperfeiçoada por Monteiro (1971). A respeito disso, Nunes e Lombardo (1995, p. 21) afirmam: "[...] embora a variabilidade seja uma componente conhecida da dinâmica climática, seu impacto, mesmo dentro de limites esperados, pode ter reflexos significativos nas mais diversas atividades humanas, como agropecuária, indústria e produção de energia [...]".

A metodologia quantitativa apresenta-nos uma complexidade a mais, pois muitas vezes o pesquisador se depara com longas séries de dados climatológicos das mais variadas fontes. Assim, em muitas pesquisas da Climatologia desprende-se muito mais tempo com cálculos e planilhas do que com aquilo que Sant'Anna Neto (2008) chamou de Geografia do Clima, interessada nas relações entre Sociedade x Clima. Neste sentido, a estatística fornece a ele possibilidades de trabalhar com um volume menor de informações aplicando-se a técnica apropriada.

\section{SOBRE TÉCNICAS ESTATÍSTICAS NA CLIMATOLOGIA}

Uma dessas técnicas foi utilizada por Barbosa (2008), buscando trabalhar com um número menor de dados, ao mesmo tempo em que buscava investigar a variabilidade das precipitações em alguns postos pluviométricos de um recorte espacial específico. $O$ pesquisador empregou uma técnica de estatística multivariada no domínio da Análise Factorial, conhecida como Análise de Componentes Principais - ACP. Segundo o autor, a técnica permite a visualização e redução de dados contidos em tabelas de grandes dimensões, cruzando certo número de indivíduos com as variáveis quantitativas que os caracterizam, reduzindo essas variáveis em um único fator representativo de um conjunto de dados. No caso da pesquisa citada, aos totais anuais de cada posto pluviométrico foi conferido um valor de carga (totais anuais), sendo que aqueles que apresentam carga maior são os mais representativos da amostra. A técnica permitiu ao pesquisador a caracterização do posto mais representativo para a pesquisa em questão, visando a aplicação de técnicas matemáticas apenas para os dados dos postos mais representativos dentro de cada um dos três recortes espaciais da pesquisa.

Como principal medida para avaliar a posição central de um conjunto de valores, o cálculo da média aritmética é o procedimento mais simples e comum passível de ser utilizado. Essa medida de tendência central expressa o somatório de todos os elementos da série dividido pelo número total de elementos. Seu cálculo é dado pela seguinte fórmula, onde $a^{1}+a^{2}+a^{3} \ldots$ são os elementos da série de dados e o valor $n$ é o número de elementos totais da série.

$$
\mu=\frac{1}{n} \sum_{i=1}^{n} a_{i}=\frac{a_{1}+a_{2}+\cdots+a_{n}}{n}
$$


Além da média, outras técnicas estatísticas são comumente empregadas para a verificação da variabilidade dos dados de elementos climáticos em relação à sua tendência central. Podemos citar o Desvio Padrão (S) e o Coeficiente de Variação (CV). O primeiro deles consiste em uma medida de dispersão, que considera a totalidade dos valores da variável em estudo. Sua fórmula é a seguinte, onde $s$ é o desvio padrão, xi é uma variável pontual da série e $\mu$ é a média das variáveis para um conjunto $n$ de dados.

$$
s=\sqrt{\frac{1}{N-1} \sum_{i=1}^{N}\left(x_{i}-\mu\right)^{2}}
$$

Porém altos índices de desvio padrão indicam maior dispersão (variância ou heterogeneidade) do conjunto de valores dos elementos climáticos da série. Entretanto, os valores elevados das variáveis brutas apresentam índices de desvio padrão igualmente altos, podendo confundir numa análise espacial comparativa, fato observado por Gerardi e Silva (1981). O desvio padrão admitiria somente uma interpretação simples e segura se a densidade de probabilidade de origem fosse considerada normal. Essa técnica perde eficiência quando as distribuições são muito assimétricas, o que pode ocorrer com variáveis climáticas. Para propiciar uma observação amenizando essa "contaminação" de montantes elevados de precipitação, é recomendado usar o Coeficiente de Variação, que indicaria a variação relativa, onde s é o desvio padrão e $\mu$ é a média.

$$
c v=\frac{s}{\mu} * 100
$$

A média e o desvio padrão, como vimos, são medidas usadas para avaliar a posição central e a dispersão de um conjunto de índices. Contudo, essas medidas são fortemente influenciadas por valores discrepantes. Pensando nisso, existe algumas possibilidades de outras técnicas estatísticas aplicadas pela Climatologia Geográfica que são menos afetadas por valores extremos.

A Técnica dos Quantis faz referência às medidas de separação para distribuições de probabilidade ou para suas amostras. Segundo Xavier, Xavier e Alves (2007), um quantil de ordem $\mathrm{p}$ (definido para $0<\mathrm{p}<1$ ) é um valor numérico que secciona a distribuição em duas partes, com probabilidades $p$ (à esquerda deste quantil "teórico") e 1-p (à direita). A vantagem do uso de quantis foi reconhecida por Galton (1822 - 1911) em 1896, quando este comparou períodos consecutivos ou anos-base distintos, com vistas à avaliação de mudanças sociais ocorridas ao longo do tempo. Esses mesmos autores assinalam que uma das vantagens do emprego dos quantis em relação ao desvio padrão é a sua capacidade para lidar com assimetrias ao descrever o fenômeno aleatório estudado como chuva, temperatura ou umidade atmosférica. 
Para Xavier (2001), um benefício do uso da técnica dos quantis se aplica à sua extrema simplicidade conceitual, aliada à uma interpretação precisa em termos probabilísticos ou das distribuições empíricas de frequências associadas, o que não ocorreria em outras técnicas. Além disso, os quantis podem ser estimados diretamente a partir dos dados empíricos, embora também possam ser determinados via um modelo teórico ajustado aos dados.

Ainda com vistas à exploração da variabilidade comportamental dos dados climáticos, costuma-se utilizar muito figuras gráficas conhecidas como Histogramas, muito presente nos livros didáticos do ensino fundamental e médio (Climogramas), que representam a distribuição de frequências de uma variável. Eles relacionam categorias de valores, juntamente com suas contagens ou frequências que se enquadram em cada categoria (TRIOLA, 1998). No histograma, a base é o limite das classes e as alturas são as frequências. A simples observação da forma do histograma nos permite fazer algumas inferências, entre elas, o grau de dispersão da variável. O uso da distribuição de frequências permite extração de informações fundamentais, como por exemplo, faixas de chuva com maiores ocorrências e vice-versa. Essa técnica é muito utilizada em pesquisas na área da engenharia civil, visando um quadro mais geral da distribuição de chuvas em determinado local.

Além das técnicas estatísticas empregadas no estudo da variabilidade, existem técnicas que buscam mapear mudanças nos padrões de distribuição temporal dos elementos climáticos. Uma das possibilidades seria usar gráficos de Médias Normalizadas, que nos permite uma comparação de valores escalares de uma variável em relação ao valor máximo e mínimo da série temporal utilizada, o que possibilita uma análise mais apurada do elemento climático, uma vez que as médias são calculadas entre dois valores definidos. As médias normalizadas - MN são calculadas com o emprego da seguinte fórmula, onde $\mu$ é a média de um conjunto de valores, xm é o valor mínimo da série e xM o valor máximo da série.

$$
m n=\frac{\mu-x_{m}}{x_{M}-x_{m}}
$$

Além das médias normalizadas, os Gráficos de Curva de Massa também podem ser utilizados na busca de mudanças em padrões comportamentais de elementos do clima. Esses gráficos representam a maneira como um dado valor vai se acumulando no decorrer de certo período de tempo. Ano após ano, as alturas vão sendo somadas, resultando em uma curva sempre crescente. Nas curvas poderemos perceber, eventualmente, inflexões positivas ou negativas, que indicariam anos nos quais o acréscimo ou decréscimo da variável em análise teria sido mais significativo. Tal técnica foi usada por Nunes (1997), no 
intuito de observar eventual mudança climática. As curvas de massa também são construídas visando identificar algumas inconsistências nos dados das estações pluviométricas a partir do ângulo de inclinação das curvas. Se estas não apresentarem mudanças abruptas de inclinação, significa que os dados são consistentes e adequados. Seções abruptas nas curvas podem indicar inconsistência dos dados provenientes, por exemplo, da mudança de lugar de determinada estação ou da falta de coleta dos dados por um período longo.

Outra técnica que pode ser utilizada para o mapeamento comportamental dos elementos é o Cálculo de Índice de Mudança, que permite salientar as diferenças no comportamento habitual dos elementos entre escalas temporais definidas. Na fórmula abaixo, ilustramos, por exemplo, como esse índice poderia ser calculado para uma comparação comportamental entre décadas, onde im é o índice de mudança decadal, $\mu 1$ é a média da década inicial; $\mu 2$ é a média da década seguinte.

$$
i m=\frac{-\left(\mu_{1}-\mu_{2}\right)}{\mu_{2}} * 100
$$

No exemplo decadal, os índices de mudança permitem verificar alterações dos montantes de chuva para as áreas de interesse do pesquisador, de sorte a identificar em quais porções do recorte espacial o elemento climático apresentou maior variabilidade intradecadal. A referida técnica pode ser utilizada em conjunto com a geoestatística de dados espaciais através da Krigagem, que visa a elaboração de mapas através da interpolação desses índices.

O comportamento dos dados climáticos pode, além de sofrer variações no decorrer do tempo, apresentar tendências. Para explorar essas tendências, pode-se utilizar a Técnica da Regressão Linear, geralmente feita sob um referencial teórico que justifique a adoção de alguma relação matemática de causalidade. Em termos estatísticos, busca-se verificar a manutenção, o aumento ou a diminuição dos valores dentro de uma sequência temporal. Baseada na técnica dos mínimos quadrados, que ajusta uma reta a um conjunto de pontos, o procedimento foi utilizado por Alnaizy e Simonet (2012), Antonini et al. (2018), Christofoletti (1992), Galina (2002), Maia (2003) e Sentelhas et al. Essa reta de $1^{\circ} \mathrm{grau}(\mathrm{y}=$ $\mathrm{ax}+\mathrm{b}$ ) é caracterizada por um coeficiente angular (a) que determina o grau de tendência da variável. Outra informação importante relacionada a essa técnica é o valor de $\mathrm{R}^{2}$ (coeficiente de determinação), que indica se a reta desenhada para a variável representa bem a realidade dos dados brutos: quanto mais próximo de 1 for o valor de $\mathrm{R}^{2}$, maior será o ajuste da reta à distribuição dos dados. Esta poderá se revelar crescente, decrescente ou estacionária, constituindo importante subsídio para se detectar mudanças climáticas, especialmente as de natureza térmica ou pluviométrica. 
Outro aspecto comum nas Pesquisas em Climatologia Geográfica é a busca do mapeamento das relações entre duas variáveis. Uma técnica conhecida como Análise de Correlação de Dados pode ser utilizada quando procuramos estudar a relação (correlação) entre duas variáveis distintas, como, por exemplo, precipitação e temperatura ou temperatura com altitude. Uma maneira de visualizarmos o correlacionamento de duas variáveis é com a elaboração de Diagramas de Dispersão, no qual os valores das variáveis são apresentados por pontos dentro de um sistema cartesiano. Cabe ressaltar que o conceito de correlação se refere a uma associação numérica entre duas variáveis, não implicando, necessariamente, uma relação de causa e efeito entre elas.

Outra técnica conhecida e bastante utilizada na pesquisa em Climatologia Geográfica é a Padronização de Dados. Essa técnica, que reflete a distribuição estatística normal padrão, é bastante usada para o cálculo de anomalias em ciências atmosféricas. Pela teoria, cerca de $2 / 3(68,3 \%)$ das ocorrências de um dado evento estão contidos entre os limites de 1 a 1 desvios padrão (s), estando dentro do habitual; 95,45\% das ocorrências estariam entre -2s a 2 s e $99,7 \%$ dos eventos estariam contidos entre -3s e 3s. Em outras palavras, o valor padronizado é uma medida relativa que nos fornece o quanto o valor de uma variável de um conjunto de dados se afasta da média, em unidade de desvio padrão. Ainda que dados referentes aos elementos climáticos dificilmente se ajustem de maneira completa a uma distribuição teórica, a padronização é amplamente usada devido à sua facilidade em destacar, com simplicidade, as excepcionalidades do fenômeno sob avaliação, como as chuvas, por exemplo. Outra vantagem no uso de uma distribuição estatística é que os valores são transformados em variáveis que refletem o seu grau de normalidade ou magnitudes dos extremos, permitindo a comparação entre dados de características diversas. Na fórmula para a padronização a seguir, z é o valor padronizado; Xi um valor único da série, $\mu$ é a média da série e $s$ é o desvio padrão da série.

$$
Z=\frac{X_{i}-\mu}{s}
$$

Como discutido até aqui, a Climatologia Geográfica utiliza-se de várias técnicas estatísticas buscando compreender o funcionamento dos elementos do clima, sua variabilidade, tendências e a presença de comportamento padrão. Várias técnicas foram descritas neste sentido. Além disso, identificamos a possibilidade de trabalhar com técnicas que são capazes de fazer a análise entre duas variáveis de elementos diferentes. Cabe agora apontar as técnicas que a Climatologia Geográfica poderá, por opção, fazer uso nos estudos de previsibilidade ou probabilidade.

Uma técnica interessante utilizada nas pesquisas em Climatologia refere-se à necessidade de saber a probabilidade de uma determinada ocorrência voltar a acontecer; 
por exemplo, uma ocorrência de chuva intensa que, por ventura, trouxe muitos impactos negativos para uma região. Qual a probabilidade de uma chuva com a mesma intensidade ocorrer novamente? Poderíamos fazer mão do cálculo de Tempo de Retorno para avaliar as incidências de extremos para um número determinado de anos, por meio de uma variável $\mathrm{K}$.

Utilizando a Teoria dos Valores Extremos para determinar vários períodos de retorno, Weiss (1955) concluiu que esta técnica fornece aproximação satisfatória na análise da distribuição dos valores extremos meteorológicos. Em outras palavras, ela estima o tempo para que um determinado evento de precipitação ocorra novamente. O cálculo do tempo de retorno é feito com a seguinte fórmula, onde tr é o tempo de retorno e $K$ os coeficientes de frequência para cada $\operatorname{tr}$ (tabela 1); $\mu$ é a média e so desvio padrão.

$$
\operatorname{tr}=\mu+K * S
$$

Tabela 1 - Variáveis para cálculo do tempo de retorno ou recorrência

Período de Recorrência

\begin{tabular}{c|c|c|c|c|c|r|r}
\hline $\operatorname{tr}$ & 5 & 10 & 15 & 20 & 25 & 50 & 100 \\
\hline$K$ & 0,866 & 1,541 & 1,917 & 2,188 & 2,393 & 3,026 & 3,653 \\
\hline
\end{tabular}

Fonte: Weiss (1955).

Alguns pesquisadores também fazem uso de técnicas estatísticas apoiadas em elementos empíricos de análise. Um exemplo interessante a respeito dessa concepção é a determinação de alturas pluviométricas consideradas extremas segundo impactos associados. Barbosa (2008) fez uso dessa possibilidade empírica para determinar alturas pluviométricas críticas na região do litoral paulista e seu entorno. Segundo o autor, por ser uma área heterogênea segundo a disposição espacial dos objetos naturais e artificiais, as alturas pluviométricas podem não causar impactos nas regiões serranas do litoral. Entretanto, a depender dos volumes, o mesmo pode trazer impactos desastrosos em áreas de forte ocupação urbana. O autor, sob essa perspectiva, elaborou as seguintes faixas de precipitação e os impactos associados:

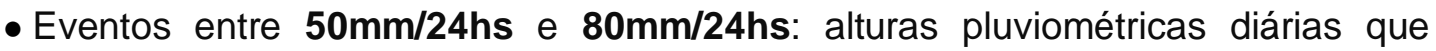
podem ou não causar algum tipo de impacto negativo.

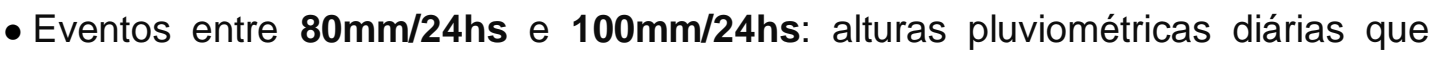
causam algum tipo de impacto negativo.

- Eventos entre $100 \mathrm{~mm} / \mathbf{2 4 h s}$ e $\mu \mathrm{mm} / \mathbf{2 4 h s : ~ a l t u r a s ~ p l u v i o m e ́ t r i c a s ~ d i a ́ r i a s ~ q u e ~}$ causam impactos negativos de média intensidade.

- Eventos acima de $\mu \mathrm{mm} / \mathbf{2 4 h s : ~ a l t u r a s ~ p l u v i o m e ́ t r i c a s ~ d i a ́ r i a s ~ q u e ~ c a u s a m ~}$ impactos negativos de forte intensidade. 
Os valores classificados como $\mu$ referem-se às médias das maiores alturas diárias listadas ano a ano segundo cada estação utilizada pelo pesquisador. Segundo Barbosa (2008), em grande parte das estações os valores superavam os $100 \mathrm{~mm} / 24 \mathrm{hs}$ de chuva, com exceção dos postos pluviométricos de Bananal e São José do Barreiro onde os valores foram $76,9 \mathrm{~mm} / 24 \mathrm{hs}$ e $81,2 \mathrm{~mm} / 24 \mathrm{hs}$, significando que valores entre $50 \mathrm{~mm} / 24 \mathrm{hs}$ e o valor possuem um peso maior na deflagração de eventos negativos nesses locais.

Um dos grandes problemas enfrentados pelos pesquisadores em Climatologia Geográfica é a ausência de estações pluviométricas ou meteorológicas em porções do território. Esse problema exige do pesquisador geógrafo um conhecimento além das disciplinas com as quais este está mais habituado, possibilitando-o contornar essa dificuldade em encontrar informações climáticas em áreas do território cujos objetos técnicos responsáveis por essa coleta estão ausentes. Um dos conhecimentos necessários para o enfrentamento dessa questão é através de técnicas de interpolação de dados, visando estimar espacialmente os índices climáticos em áreas cuja caraterística principal é a ausência da informação climática.

Outra questão limitante nas pesquisas em Climatologia Geográfica, além da ausência de dados meteorológicos, refere-se à própria escala de registro desses dados. Em relação aos postos pluviômetros, esses aparelhos nos fornecem informações a respeito das precipitações segundo escalas diárias, medindo a altura da precipitação no período de 24 horas. Infelizmente, a rede pluviográfica capaz de fornecer informações sobre chuvas para escalas menores que a diária é bastante reduzida. Isso caracteriza um fator limitante para as pesquisas que se concentram, por exemplo, no estudo de chuvas intensas.

Visando contornar este problema, Torrico (1974), ao plotar em um papel de probabilidades as precipitações de 24 horas e de 1 hora para diferentes estações pluviográficas do Brasil, verificou que, prolongando as respectivas semi retas de altura de precipitação por duração, estas tendem a cortar o eixo das abscissas em um mesmo ponto para determinadas áreas geográficas. Esta tendência significa que, em cada área homóloga, a relação entre as precipitações de 1 hora e de 24 horas para um mesmo tempo de recorrência é constante e independe das alturas de precipitação registradas.

Sendo assim, esta relação constante nos permite determinar a correlação entre os dados das estações pluviométricas para chuvas de durações inferiores a 24 horas. Esse trabalho é conhecido como Método das Isozonas, que é utilizado para estimar alturas de chuvas com durações inferiores a 24 horas. Recebe este nome porque dividiu o mapa do Brasil em oito áreas homólogas denominadas isozonas (figura 1).

- As isozonas B e C tipificam a zona de influência marítima com coeficientes de intensidades suaves; 
- As isozonas E e F são exemplos de zona continental e do noroeste, respectivamente, com coeficientes de intensidades altos;

- A isozona D é uma zona de transição entre a zona continental e a zona marítima. Esta isozona se prolonga, caracterizando a zona de influência do Rio Amazonas;

- As isozonas G e H são zonas de caatinga nordestina, possuindo coeficientes de intensidade muito altos;

- A isozona A coincide com a zona de maior precipitação anual do Brasil, com coeficientes de intensidade baixos.

Figura 1 - Áreas de Isozonas para o Brasil

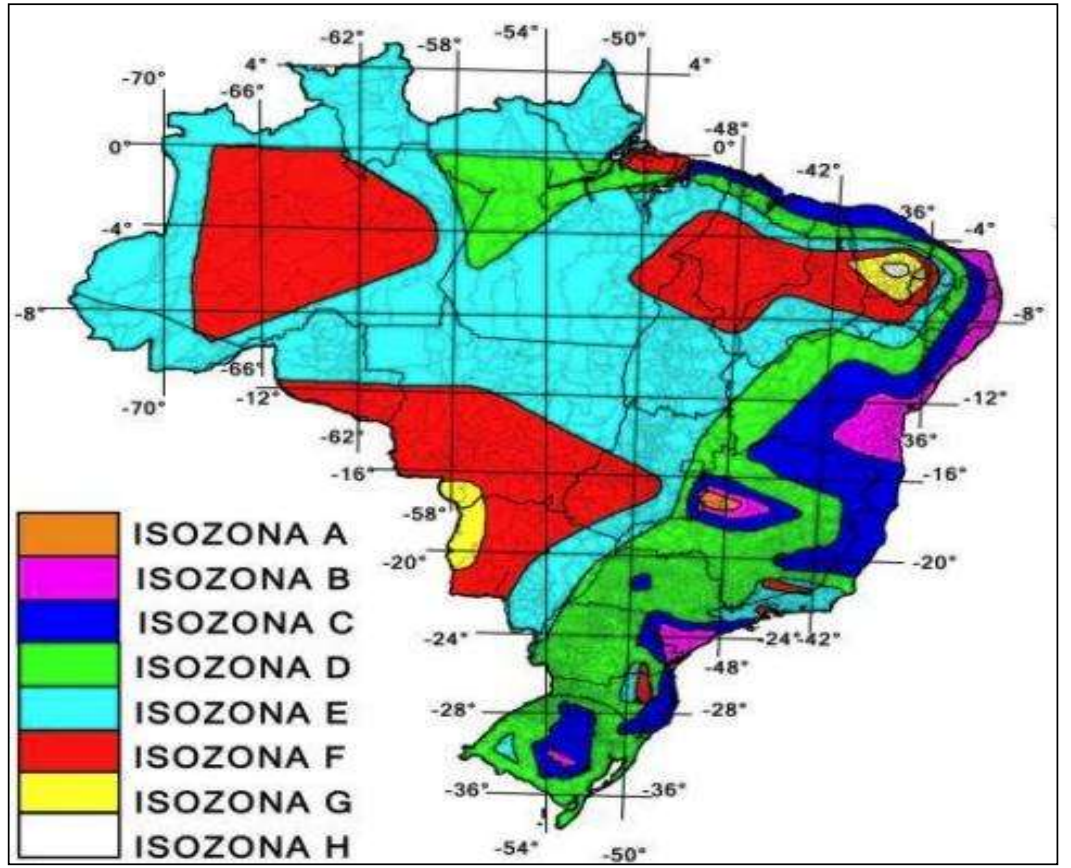

Fonte: Torrico, 1974.

Assim, em uma determinada região, os dados pluviométricos coletados em uma estação poderiam ser utilizados para obter as chuvas intensas para diferentes tempos de retorno segundo metodologia própria.

O trabalho de espacialização dos coeficientes de relação entre durações de chuvas intensas apresentado por Torrico (1974), foi pioneiro no estudo da extrapolação de chuvas intensas para locais onde, antes, não havia nenhuma estação meteorológica ou posto pluviométrico. Havia uma grande ausência de informação referente a dados climáticos em escala menor que a diária. Porém, nos dias atuais, com o incremento da malha de pluviógrafos cobrindo quase todo território nacional, a falta de atualização dessas séries históricas por registros mais recentes sobre as precipitações intensas torna questionável a sua aplicação. 
Pensando nessa desatualização, Basso et al. (2016) realizaram uma pesquisa visando uma revisão dos coeficientes de relação entre duração de chuvas intensas, utilizando das informações registradas por aproximadamente 1.000 pluviógrafos, lembrando que, na elaboração de Torrico, este utilizou-se de 98 fontes de informações, entre pluviógrafos e postos pluviométricos, a maioria localizando-se na região Sul e Sudeste do país, enquanto nas outras regiões os postos utilizados, em sua maioria, concentravam-se nas capitais ou seja, trata-se de uma atualização de grande monta.

Os pesquisadores observaram através do tratamento dos dados e da espacialização dos atuais padrões de relação entre as chuvas que, ainda que o trabalho de Torrico (1974) consiga captar as diferenças regionais de chuvas intensas, para algumas regiões as diferenças encontradas para os coeficientes foram significativas (quase $50 \%$ em algumas situações). Assim, os pesquisadores elaboraram um novo mapa de isozonas com as respectivas tabelas de coeficientes de relações entre durações atualizadas para o período de recorrência de dez anos (figura 2).

Figura 2 - Comparação da atualização das áreas de Isozonas para o Brasil
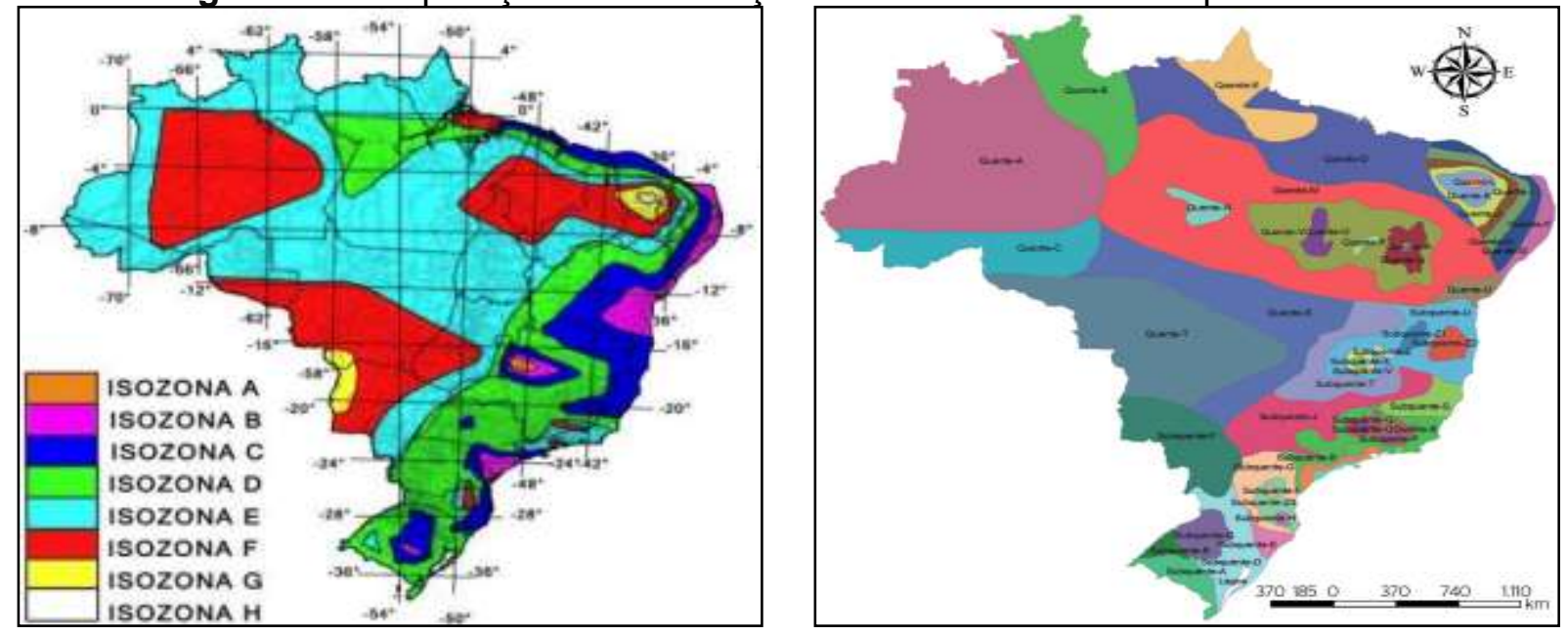

Fonte: Basso et al. (2016) e Torrico (1974).

Embora os resultados apresentados neste estudo sejam atualizados e contem com uma maior representatividade de postos e de séries temporais, é importante ressaltar que, particularmente, no Centro-Oeste e no Norte do Brasil, a densidade de postos pluviométricos ainda é baixa.

Essas foram algumas das técnicas utilizadas nos tratamentos dos registros climáticos em pesquisas na área de Climatologia Geográfica. Percebe-se a grande influência da estatística no método dinâmico da Climatologia (figura 3). O conhecimento a respeito do tratamento de variáveis de característica aleatória, como são os elementos do clima, se faz de grande importância para a formação do geógrafo climatólogo. 
Figura 3 - Organograma do universo da abordagem climática desenhada após descrição das técnicas estatísticas

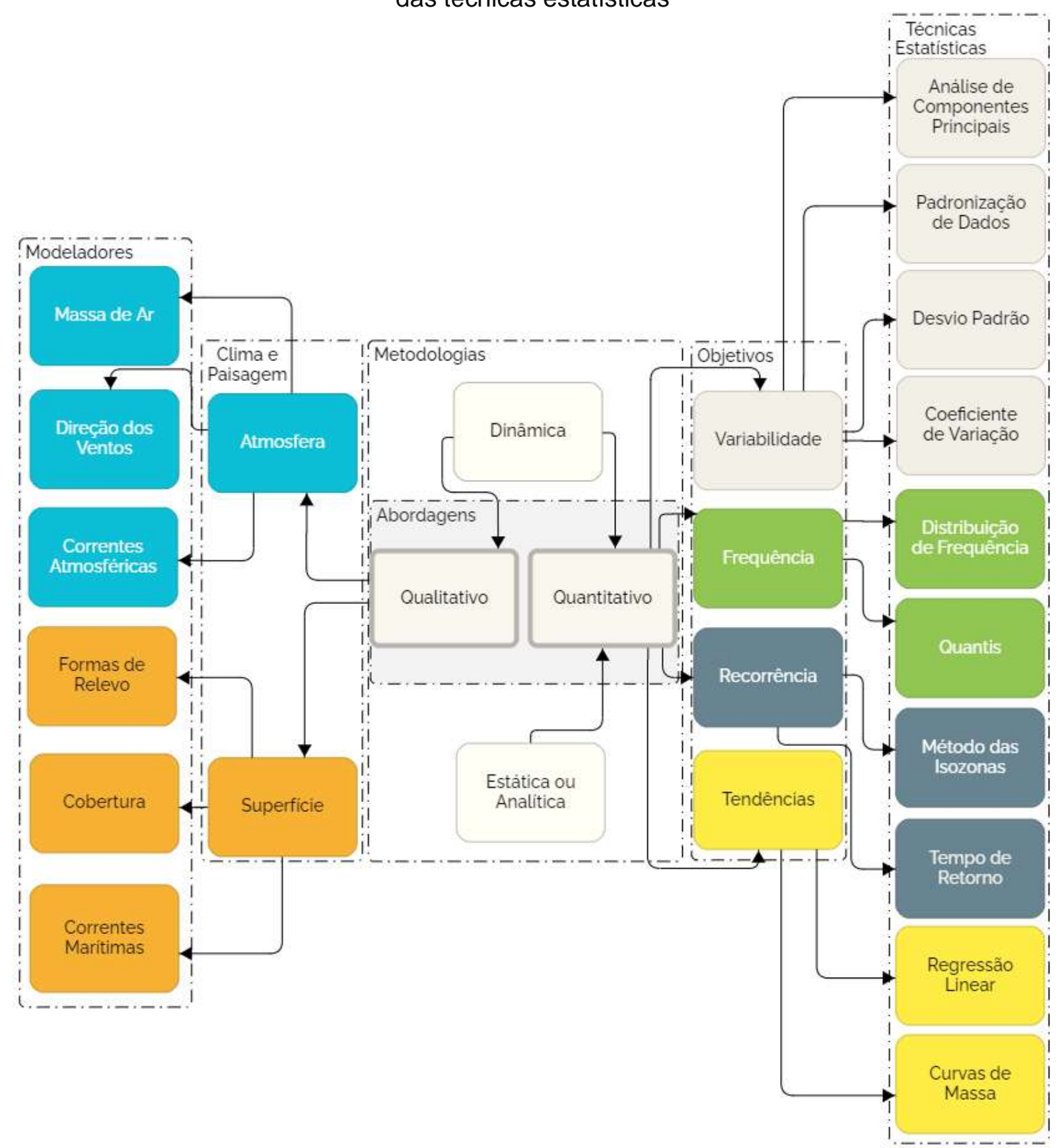

Fonte: os próprios autores.

Outra consideração que vale observar diz respeito à importância da compreensão da componente escalar nos estudos do clima. A primeira componente refere-se às escalas temporais de análise do clima. Dependendo do objetivo da pesquisa proposta, as respostas às questões serão conhecidas apenas em uma escala temporal diária, como nos exemplos dos estudos que abordam os impactos advindos de eventos extremos de precipitação. Em outros casos, como, por exemplo, na análise de estudos do solo e impactos advindos de erosões, a escala temporal se concentra nos estudos de chuvas intensas, ou seja, 
necessitamos de dados em escala menor do que 24 horas. Por outro lado, a escala mensal ou anual de análise já seriam o suficiente para a elaboração de um cenário mais geral a respeito do comportamento do clima em determinada região. Enfim, a escala de tempo está intimamente ligada ao objetivo proposto pelo pesquisador.

A segunda componente de a questão escalar em clima refere-se a escala espacial, ou seja, refere-se aos elementos da dinâmica atmosférica responsáveis pela geração do cenário climático estudado. Essa segunda componente é mais complexa de compreensão, pois envolve abstrações e um entendimento a respeito do funcionamento de certos sistemas físicos. Além disso, trata-se de componentes climáticas que afetam os elementos em múltiplas escalas. Por isso, nosso próximo item tratará da importância e da explicação em Climatologia Geográfica da escala espacial dos fenômenos climáticos em suas relações e interrelações.

\section{AS RELAÇÕES MULTI - ESCALARES DO FENÔMENO CLIMÁTICO}

Os sistemas atmosféricos são dinâmicos por natureza, seguindo um ritmo que pode sofrer variações. Segundo Nunes (1998), a compreensão da escala nos estudos da Climatologia Geográfica é de extrema importância para a compreensão dos diferentes impactos adversos da variabilidade do clima, característica essa intrínseca dos elementos climáticos. Portanto, nos estudos da Climatologia Geográfica, o entendimento do funcionamento da dinâmica atmosférica nas mais variadas escalas visa compreender o ritmo atmosférico em sua totalidade, segundo paradigma proposto por Monteiro (1971). Neste sentido, a necessidade da compreensão multi-escalar do fenômeno é um dos aspectos mais importantes da pesquisa em Climatologia.

A integração de fenômenos em um dado tempo e espaço criaria unidades, sendo que a abordagem da pesquisa em clima dependerá do nível escalar de análise - extensão espacial e duração do fenômeno. Os processos físicos que interagem nas escalas superiores refletem essas relações em escalas inferiores, modificando sucessivamente o comportamento da atmosfera, porém repercutindo de forma limitada no sentido contrário. Colocado esses critérios, Ribeiro (1993) cria uma taxonomia baseada em três níveis interativos de grandeza. O quadro 1 ilustra a classificação de acordo com as interações que definiriam os três níveis escalares da pesquisa climática: macroclimático, mesoclimático e microclimático.

Dentro das ordens de grandeza propostas, existem zonas climáticas definidas segundo sua extensão horizontal. A de clima zonal corresponde a circulação geral da atmosfera em uma extensão horizontal entre 1.000 e 5.000 quilômetros. Sua extensão 
vertical abrange toda a atmosfera. Nessa escala, a configuração climática teria uma duração mínima de uma semana até seis meses.

Quadro 1 - Escalas climáticas espaciais e interações

\begin{tabular}{|c|c|c|}
\hline Macroclimático & Mesoclimático & Microclimático \\
\hline $\begin{array}{c}\text { Interação entre radiação solar, } \\
\text { curvatura da terra e movimentos } \\
\text { de rotação e translação. }\end{array}$ & $\begin{array}{c}\text { Interação entre energia } \\
\text { disponível para processos de } \\
\text { evaporação e geração de } \\
\text { campos de pressão e feições do } \\
\text { meio terrestre. }\end{array}$ & $\begin{array}{c}\text { Interação entre sistemas } \\
\text { ambientais particulares na } \\
\text { modificação dos fluxos de } \\
\text { energia, umidade, massa e }\end{array}$ \\
\hline
\end{tabular}

Fonte: Ribeiro (1993).

A configuração climática na escala regional tem sua origem na ação modificadora da circulação geral da atmosfera em interação com os fatores superficiais que engendram os centros de ação. Possui uma extensão horizontal entre 150 e 2.500 quilômetros e vertical com limites determinados entre a superfície terrestre e a tropopausa - limite superior da troposfera. Nessa escala climática os sucessivos estados de tempo atmosférico durariam de um a 30 dias.

O clima local ou mesoclima seria moldado pela variabilidade no interior do clima regional, muito devido às diferentes feições fisiográficas da paisagem, destacando-se o relevo. A ação antrópica na alteração da cobertura do solo e na composição da atmosfera também geram alterações no clima regional, influenciando assim o caráter climático local.

Outras escalas são o topoclima e o microclima, gerado pela amplitude das trocas de gases e energia entre as feições particulares da superfície terrestre (rugosidades) e o ar que as envolve, originando novas configurações climáticas dentro do clima local. Se alterações no uso da terra interferem em todos os sistemas do planeta - em especial na composição da atmosfera, afetando o equilíbrio radiante - todas as escalas de maior detalhe serão afetadas.

Em todas as escalas até aqui definidas existem fenômenos particulares que influenciam o comportamento dos elementos do clima. Na escala global e regional, esses fenômenos influenciam a variabilidade ano a ano do comportamento climático padrão, como por exemplo o El Niño. Como exemplo dessa variabilidade influenciada por fenômenos de grande escala, Nunes e Calbete (2000) realizaram um estudo na região do Vale do Paraíba, onde avaliaram se os padrões de precipitação apresentaram mudanças que pudessem ser responsáveis pelo aumento de eventos de deslizamento e alagamentos observados na área. Concluíram que o outono e o inverno apresentaram aumento nos totais pluviométricos no período entre 1978 e 1997, relacionado esse aumento à atuação do fenômeno do El Niño.

Outros fatores como anomalias da Temperatura da Superfície do Mar - TSM no Atlântico e o deslocamento da Zona de Convergência do Atlântico Sul - ZCAS são exemplos 
de fenômenos em grande escala que impactam diretamente o tempo atmosférico, desencadeando uma série de efeitos sobre as escalas inferiores do clima e sobre a categoria geográfica do lugar.

A ZCAS é convencionalmente definida como uma persistente faixa de nebulosidade orientada no sentido Noroeste-Sudoeste, que se estende do Sul da Amazônia ao Atlântico Sul - Central, compreendendo alguns milhares de quilômetros, bem caracterizada nos meses de verão. Estudos mostram um importante papel das ZCAS na transferência de calor e umidade dos trópicos para as latitudes mais altas. As observações feitas a respeito do comportamento dinâmico das ZCAS indicam uma associação entre períodos de enchentes de verão na Região Sudeste e veranicos na Região Sul com permanência das ZCAS por períodos prolongados sobre a Região Sudeste. Por outro lado, quando as ZCAS se estabilizam mais ao Sul observa-se veranicos no Sudeste e enchentes na Região Sul.

A ZCAS se alinha com uma região de forte gradiente de TSM. Esse alinhamento acarreta uma forte influência dessas temperaturas sobre o comportamento da ZCAS, porém é possível que as anomalias de temperatura presentes na superfície sejam somente uma resposta à anomalia de vento na superfície do oceano, originária da própria ZCAS. Uma vez estabelecida a circulação padrão da ZCAS é razoável supor que haja uma realimentação positiva entre a circulação atmosférica e as anomalias de TSM ancorando as configurações na atmosfera e no oceano.

Os fenômenos atmosféricos ocorrem em um largo espectro nas escalas temporoespaciais. A compreensão da variabilidade no tempo e no espaço dos fenômenos atmosféricos em sua relação com outros fatores do meio físico e socioeconômico nos fornece um entendimento a respeito dessa dinâmica. O importante é a compreensão de que o tempo e o espaço são dimensões indissociáveis em estudos da Climatologia Geográfica.

A escala reflete um comportamento dinâmico e não uma média estática. As escalas de maior abrangência não são a mera soma dos fenômenos de menor abrangência. Em níveis escalares menores, os componentes locais são alterados pelas variáveis desse nível e por aqueles da circulação regional e zonal. Fenômenos ocorrentes em escalas diversas estão ligados e entrelaçados a partir de vários mecanismos de retroalimentação, criando condições específicas que precisam ser avaliadas pelo pesquisador em Climatologia.

Sobre esse relacionamento de retroalimentação, Ayoade (1986) estabelece escalas de circulação atmosférica por critérios de sua extensão espacial, reforçando a importância dos movimentos atmosféricos em escala global e sua atuação direta sobre as escalas inferiores regionais e locais. Segundo a nomenclatura de Ayoade (1986), teríamos a escala de circulação primária, secundária e terciária em ordem decrescente de grandeza, tanto em escalas de área quanto de tempo. 
A circulação primária corresponderia à circulação geral da atmosfera, descrita por Barry e Chorley (1976, p. 76) como sendo "[...] os padrões em larga escala, ou globais, de vento e pressão que se mantêm ao longo do ano ou se repetem sazonalmente". Segundo Ayoade (1986), essa circulação geral é que determina o padrão dos climas do mundo. Inseridos dentro da circulação geral estão os sistemas circulatórios secundários (depressões, anticiclones...) que se movem rapidamente e possuem uma existência breve. Por fim, os sistemas de circulação terciária que consistem principalmente de sistemas de ventos locais, tais como as brisas terrestres e marítimas. São sistemas que possuem localização específica, sendo controlados por fatores locais. Seus períodos de duração são mais curtos do que os dos sistemas de circulação secundária e primária.

Conforme salientamos, a atmosfera encontra-se em contínua dinâmica. Dentro dessa dinâmica temos elementos que podem ser tratados em diferentes escalas de interesse. A atmosfera e outros componentes do sistema ambiental - homem inclusive - integram-se, interagindo numa sucessão de eventos que se cristalizam no espaço. Para entendermos a dinâmica atmosférica de uma área temos que começar com uma visão global da localidade em que ela está inserida. Para isso, começa-se com os controles de grande escala ou circulação primária, passando pelas perturbações de meso-escala (regional ou circulação secundária), chegando por fim nas condições locais, resultado da interação dinâmica de diversas naturezas (botânicas, climático-botânicas, outras).

Feito esse entendimento breve a respeito das relações multi-escalares dos fenômenos climáticos, cabe, portanto, aos pesquisadores em Climatologia Geográfica uma visão holística-escalar (figura 4) e dialética-escalar, na qual sociedade e clima se relacionam como vetores das mudanças climáticas e sociais. 
Figura 4 - Organograma para compreensão de a questão escalar na Climatologia Geográfica

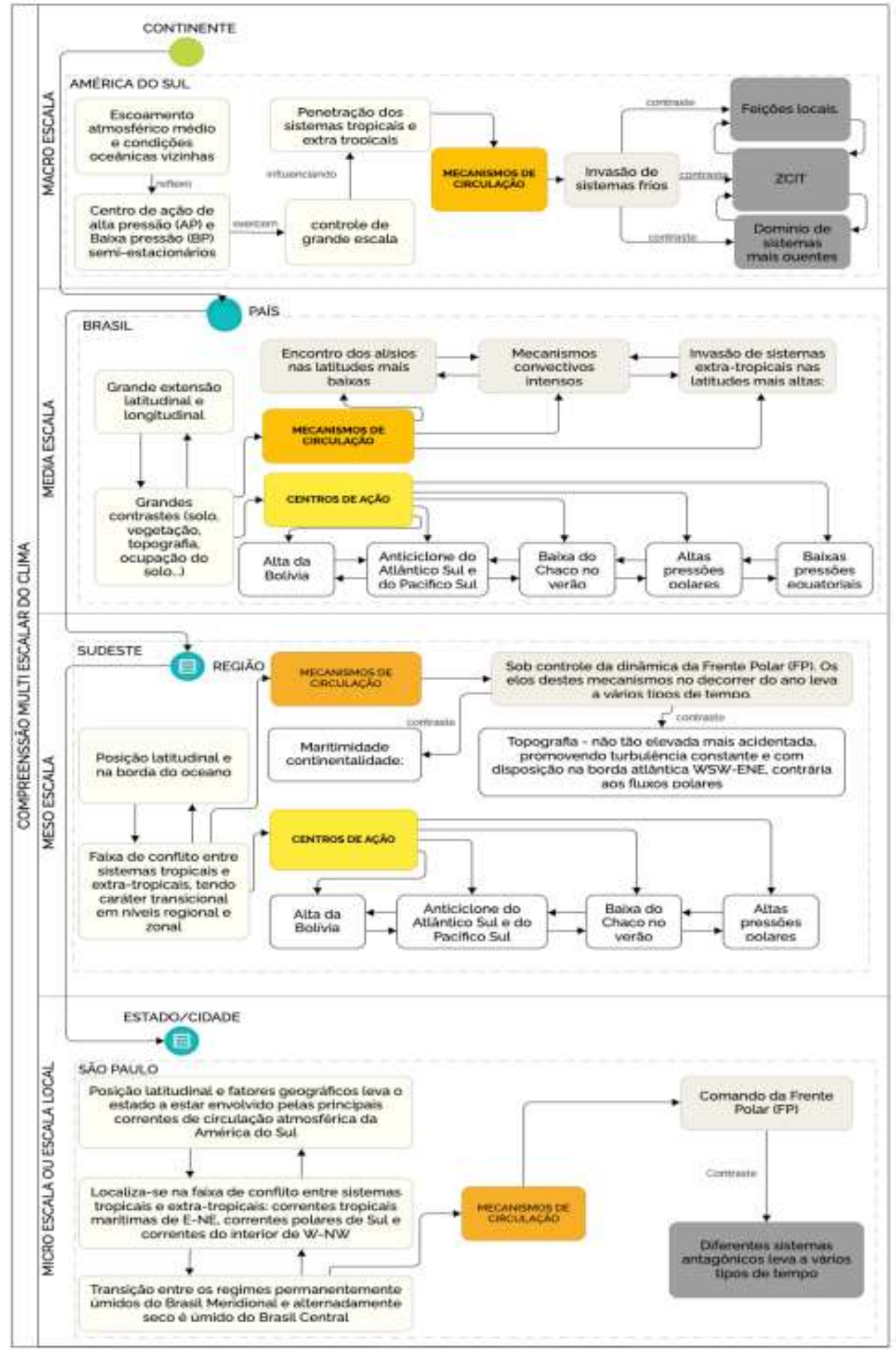

Fonte: os próprios autores.

\section{CONSIDERAÇÕES FINAIS}

Frente à discussão realizada, observamos que a Climatologia Geográfica faz uso de uma série de ferramentas ou instrumentos estatísticos na direção de compreender 
matematicamente o comportamento do fenômeno climático dentro das suas mais variadas características: variabilidade, tendências, frequência e recorrência, entre outras. Mas essa compreensão será sempre parcial caso a visão multi-escalar do fenômeno não seja considerada na análise climática realizada. O fenômeno climático é, portanto, matemático e multi-escalar, o que nos remete à importância do recorte tanto espacial quanto temporal na análise a ser realizada.

\section{REFERÊNCIAS}

ANTONINI, Jorge César dos Anjos et al. Modelo matemático para estimativa da temperatura média diária do ar no Estado de Goiás. Pesquisa Agropecuária Brasileira, Brasília, v. 44, n. 4, p. 331-338, 2009. Disponível em: https://doi.org/10.1590/S0100-204X2009000400001. Acesso em: 15 set. 2021.

ALNAIZY, Raafat; SIMONET, Daniel. Analysis of water evaporation and drift losses during irrigation in semi-arid areas of Sharjah (UAE) and Riyadh (KSA). Natural Resources

Research, New York, v. 21, n. 2, p. 285-299, 2012. Disponível em: http://dx.doi.org/10.1007/s11053-012-9173-5. Acesso em: 16 set. 2021.

AYOADE, Jo. Introdução à climatologia para os trópicos. Rio de Janeiro: Bertrand Brasil, 1986.

BARBOSA, João Paulo Macieira. Avaliação de técnicas empíricas e estatísticas de identificação de extremos de precipitação para o litoral paulista e entorno. 2008. Dissertação (Mestrado em Geografia) - Instituto de Geociências, Universidade Estadual de Campinas, Campinas, 2008.

BARROS, Juliana Ramalho; ZAVATTINI, João Afonso. Bases conceituais em climatologia geográfica. Mercator, Fortaleza, v. 8, n. 16, p. 255-261, 2009. Disponível em:

https://doi.org/10.4215/RM2009.0816.0019. Acesso em: 02 jun. 2021.

BARRY, Roger; CHORLEY, Richard. Atmosphere, weather and climate. London: Methuen Publishing Ltd, 1976.

BASSO, Raviel Eurico et al. Revisão das isozonas de chuvas intensas do Brasil.

Engenharia Sanitária e Ambiental, Rio de Janeiro, v. 21, n. 4, p. 635-641, 2016.

Disponível em: https://doi.org/10.1590/S1413-41522016133691. Acesso em: 04 nov. 2020.

CHRISTOFOLETTI, Antônio. Procedimentos de análise utilizados no estudo da precipitação.

Revista Geociências, São Paulo, v.11, n. 1, p. 75-98,1992.

GALINA, Márcia Helena. Mudanças climáticas de curto prazo: tendência dos regimes térmicos e hídricos e do balanço hídrico nos municípios de Ribeirão Preto, Campinas e Presidente Prudente (SP) para o período de 1969-2001. 2002. Dissertação (Mestrado em Geografia) - Instituto de Geociências e Ciências Exatas, Universidade Estadual Paulista, Rio Claro, 2002.

GERARDI, Lucia Helena de Oliveira; SILVA, Barbara-Christine Nentwig. Quantificação em Geografia. São Paulo: DIFEL, 1981.

MAIA, Diego Corrêa. Variabilidade climática e a produtividade do milho em espaços paulistas. 2003. Dissertação (Mestrado em Geografia) - Instituto de Geociências e Ciências Exatas, Universidade Estadual Paulista, Rio Claro, 2003.

MONTEIRO, Carlos Augusto de Figueiredo. Análise rítmica em climatologia. Climatologia, São Paulo, n. 1, p. 1-21, 1971. 
NUNES, Luci Hidalgo; LOMBARDO, Magda Adelaide. A questão da variabilidade climática: uma reflexão crítica. Revista do Instituto Geológico, São Paulo, v. 16, n. 1/2, p. 21-31, 1995. Disponível em: https://www.infraestruturameioambiente.sp.gov.br/wpcontent/uploads/sites/233/2012/03/16_1-2_2.pdf. Acesso em: 21 set. 2021.

NUNES, Luci Hidalgo. Distribuição espaço-temporal da pluviosidade no Estado de São Paulo: tendências, variabilidade, processos intervenientes. 1997. Tese (Doutorado em Engenharia dos Transportes) - Faculdade de Engenharia de Transportes, Universidade de São Paulo, São Paulo, 1997.

NUNES, Luci Hidalgo. A escala nas ciências atmosféricas. Revista do Instituto Geológico, São Paulo, v. 19, p. 71-73, jan./dez. 1998.

NUNES, Luci Hidalgo; CALBET, Nuri Oyamburu de. Variabilidade pluviométrica no Vale do Paraíba Paulista. In: CONGRESSO BRASILEIRO DE METEOROLOGIA, 11., 2000, Rio de Janeiro. Anais [...]. Rio de Janeiro: Sociedade Brasileira de Meteorologia, 2000. p.39873994.

RIBEIRO, Antônio Giacomini. As escalas do clima. Boletim de Geografia Teorética, Rio Claro, n. 23, p-288-294, 1993.

SANT'ANNA NETO, João Lima. Da climatologia geográfica à geografia do clima: genêse, paradigmas e aplicações do clima como fenômeno geográfico. Revista da Anpege, João Pessoa, n. 4, p.51-72, 2008. Disponível em: https://doi.org/10.5418/RA2008.0404.0004. Acesso em: 17 set. 2021.

SENTELHAS, Paulo César et al. Um século de desmatamento: efeitos no regime térmico, pluvial e no balanço hídrico em Campinas, SP. Revista Brasileira de Agrometeorologia, Santa Maria, v. 2, p.99-103, 1994.

SIQUEIRA, Beatriz. Variabilidade, distribuição e concentração da precipitação na bacia hidrográfica do Rio Paraná. 2019. Tese (Doutorado em Geografia) - Instituto de Geociências, Universidade Estadual de Campinas, Campinas, 2019.

SOARES, Sérgio Aurélio Ferreira. Spatial interpolation and geostatisc simulation with the incremental gaussian mixture network. 2016. Dissertação (Mestrado em Ciência da Computação) - Universidade Federal de Santa Catarina, Florianópolis, 2016. Disponível em: https://repositorio.ufsc.br/xmlui/handle/123456789/178581. Acesso em: 18 set. 2021.

SORRE, Max. Les fondements de la géographie humaine. Paris: Armand Colin, 1951. TORRICO, José Jaime Taborga. Práticas hidrológicas. Rio de Janeiro: Transcon, 1974. TRIOLA, Mário F. Introdução à estatística. Rio de Janeiro: LTC, 1998.

XAVIER, Teresinha de Maria Bezerra S. Tempo de Chuva: estudos climáticos e de previsão para o Ceará e Nordeste do Brasil. Fortaleza: ABC, 2001.

XAVIER, Teresinha de Maria Bezerra S.; XAVIER, Airton Fontenele Sampaio; ALVES, José Maria Brabo. Quantis e eventos extremos: aplicações em ciências da terra e ambientais. Fortaleza: RDS, 2007.

WEISS, Leonard L. A nomogram based on the theory of extreme values for determining values for various return periods. Monthly Weather Review, Boston, v. 83, n. 3, p. 69-71, 1955. 